\title{
EDITORIAL
}

\section{Basic liver immunology}

\author{
Bin Gao
}

Cellular \& Molecular Immunology (2016) 13, 265-266; doi:10.1038/cmi.2016.9; published online 4 April 2016

The liver is the largest solid organ in the body and has many unique immunological properties, including induction of immune tolerance, strong innate immunity, poor adaptive immune response versus overreactive autoimmunity and hematopoiesis in the fetal liver. Thus, the liver has been proposed as 'an immunological organ'. Although the primary functions of the liver are not traditionally considered to be immunological, the liver also performs many essential immune tasks. For example, hepatocytes are responsible for the production of $80-90 \%$ of the circulating innate immunity proteins in the body, and the liver contains a large number of resident immune cells. Therefore, researchers have also proposed that the liver is 'an innate immune organ', 'an immune organ'3 and 'a lymphoid organ'.4,5 I am honored and excited to present this special issue that contains eight excellent review articles about the current knowledge of these fascinating immunological features of the liver. The first article by Robinson et al. ${ }^{6}$ provides an overview of liver immunology and its role in liver inflammation and homeostasis. The following two papers discuss the special properties of antigen-presenting cells in the liver and their contribution to the hepatic tolerogenic effect. ${ }^{7,8}$ The last six articles explore

Laboratory of Liver Diseases, National Institute on Alcohol Abuse and Alcoholism (NIAAA), National Institutes of Health (NIH), Bethesda, MD 20892, USA

Correspondence: B Gao, MD, PhD, Laboratory of Liver Diseases, National Institute on Alcohol Abuse and Alcoholism (NIAAA), National Institutes of Health (NIH), 5625 Fishers Lane, Bethesda, MD 20892, USA.

E-mail: bgao@mail.nih.gov

Received: 27 January 2016; Accepted: 27 January 2016 hepatic innate immunity ${ }^{9-13}$ and adaptive immunity. ${ }^{14}$

Liver immune tolerance was first observed in porcine liver allo-transplantation in the 1960s and was later found to be indispensable for the maintenance of oral tolerance toward food and bacterial antigens from the gut. Over the past several decades, the immune tolerogenic function of the liver has been extensively investigated by many groups, but the underlying mechanisms still remain obscure. Evidence suggests that livermediated immune tolerance requires the complex interaction of hepatocytes, liver nonparenchymal cells and immune cells. In this special issue, two articles discuss the potential cellular and molecular mechanisms through which immune tolerance occurs in the liver. Horst et al. ${ }^{7}$ summarize the characteristics of several types of conventional and nonconventional antigen-presenting cells, as well as regulatory innate immune cells in the liver, which may have an important role in inducing immune tolerance. Grakoui and Crispe $^{8}$ also summarize many unique features of antigen-presenting cells that present hepatocellular antigens in the liver and discuss their potential role in mediating the hepatic tolerogenic effect.

The liver is constantly exposed to a wide variety of bacterial products, environment toxins and food antigens. To efficiently and rapidly protect against these potentially toxic agents without generating detrimental immune responses, the liver depends on its strong innate immune system, acting as an important innate immunity organ. ${ }^{2}$ In this special issue, five articles update and discuss the innate immunity in the liver. First, Zhou et al. ${ }^{9}$ provide evidence supporting the role of hepatocytes as a key cell type of innate immunity, as hepatocytes are responsible for

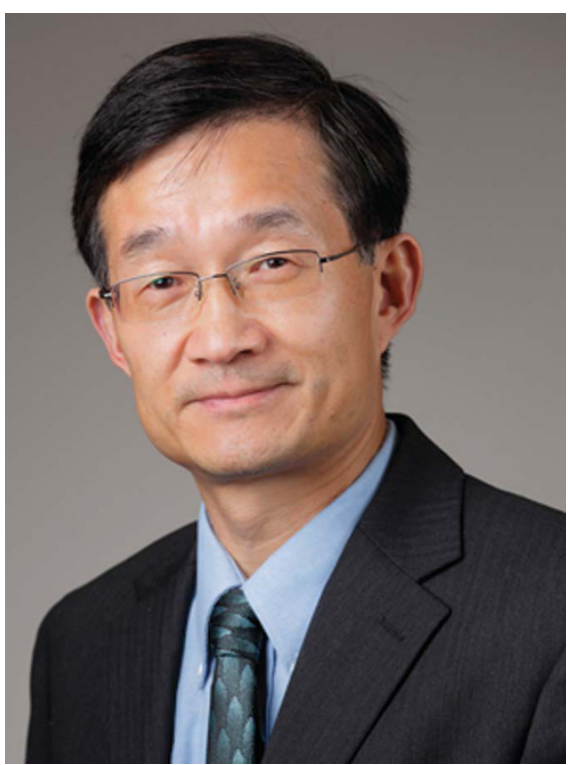

Bin Gao

the production of $80-90 \%$ of the circulating innate immunity proteins in the body, including acute phase proteins, complements, bactericidal proteins, opsonins and so on. These innate immunity proteins produced by hepatocytes have a key role in the control of both hepatic and systemic bacterial infections. In addition, Zhou et al. ${ }^{9}$ also discuss several signaling pathways and liver-enriched transcription factors that control the expression of these innate immunity proteins. Second, the liver contains the single largest population of macrophages (resident Kupffer cells), which accounts for $80-90 \%$ of the total population of fixed tissue macrophages in the body, and have a key role in eliminating insoluble waste by phagocytosis. In addition to Kupffer cells, the liver also contains a large number of infiltrating bone marrow-derived monocytes/macrophages, especially during liver injury and infection. 
In this issue, $\mathrm{Ju}$ and $\mathrm{Tacke}^{10}$ discuss the differences between liver-resident Kupffer cells and infiltrating macrophages, and summarize their functions in the maintenance of hepatic homeostasis and their involvement in both promoting and resolving liver inflammation, injury and fibrosis. Third, the liver is also enriched with another type of important innate immune cells, natural killer (NK) cells. Liver NK cells were first described as 'pit cells' in the rat liver by Wisse et al. ${ }^{15}$ in the 1970s; the pit cells were named so because they contained highly characteristic cytoplasmic granules resembling 'fruit pits' when observed with electronic microscopy. More recently, by using flow cytometry analysis of different cell surface markers, Tian's group showed, for the first time, that liver NK cells exist in two subsets including liver-resident NK cells and infiltrating NK cells. ${ }^{16}$ In this special issue, we are fortunate to have Dr Wisse, Dr Tian and their colleagues to discuss liver 'pit cells' and NK cell subsets, and to highlight recent progress in liver-resident NK cells and their functions. Fourth, NKT cells, which are also enriched in liver lymphocytes, are a heterogeneous group of $\mathrm{T}$ lymphocytes that recognize the lipid antigens presented by the nonclassical MHC class I-like molecule CD1. Because they rapidly produce a variety of cytokines after activation, NKT cells not only have an important role in the control of innate immunity but also act as a functional bridge between innate immunity and adaptive immunity. Recent studies suggest that NKT cells act as a critical regulator of the pathogenesis of liver disease by modulating liver inflammation, injury, fibrosis and regeneration, which are outlined in the review by Bandyopadhyay et al. ${ }^{12}$ Moreover, they also discuss NKT subsets and their functions in the maintenance of immune tolerance and inflammatory diseases in the liver. ${ }^{12}$ Fifth, liver sinusoidal endothelial cells are an important component of innate immunity. They are the most efficient endocytic cells in the body because they remove soluble macromolecular and colloidal waste (smaller than $100 \mathrm{~nm}$ ) from the circulation by endocytosis. In addition to these extraordinary scavenger functions, liver sinusoidal endothelial cells possess potent innate immune functions, serving as sentinel cells that detect microbial infection through pattern recognition receptor activation and (cross)-present antigens.
All of these functions are comprehensively summarized in the review article written by Knolle and Wohlleber ${ }^{13}$ in this issue.

In contrast to the strong innate immunity, adaptive immunity in the liver is less active, and the liver is an important site of T-cell tolerance induction, as demonstrated by the spontaneous acceptance of liver allografts, the persistence of some liver pathogens (viral hepatitis $B$, viral hepatitis $C$, and malaria) and the induction of oral tolerance to food antigens. However, under certain conditions, the liver also develops an uncontrolled autoreactive immune response, resulting in autoimmune liver disease. In this special issue, Gershwin and colleagues present the current understanding of adaptive immunity in the liver and discuss immunosuppression (such as in chronic viral hepatitis B or C infections and liver cancer) versus over-reactive adaptive immunity (such as autoimmune liver disease) in the liver. ${ }^{14}$

Owing to space constraints, many important questions about basic liver immunology have not been addressed in this special issue. First, although the mechanisms underlying liver immune tolerance were highlighted, those pertaining to liver transplantationassociated immune tolerance, such as hematopoietic chimerism, were not discussed. Second, peripheral blood $\mathrm{T}$ cells are composed of $95 \% \alpha \beta \mathrm{T}$ cells and $5 \% \gamma \delta \mathrm{T}$ cells, whereas liver $\mathrm{T}$ cells consist of $15-25 \%$ $\gamma \delta \mathrm{T}$ cells, and the liver is one of the richest sources of $\gamma \delta \mathrm{T}$ cells in the body. However, the function of $\gamma \delta \mathrm{T}$ cells in liver pathophysiology remains largely unknown. Third, the liver has been proposed as a lymphoid organ, and it was reported that adult livers contain hematopoietic stem cells; ${ }^{17}$ however, it is not clear whether these stem cells are responsible for the production of liverresident immune cells, such as Kupffer cells, and liver-resident NK cells. Fourth, hematopoietic stem cells are produced during embryonic development; they subsequently colonize the fetal liver, the spleen and finally the bone marrow. How the fetal liver environment regulates hematopoietic stem cells is poorly understood. In addition, the adult liver environment likely has a key role in regulating the unique distribution and functions of lymphocytes in the liver; however, the molecular mechanism remains largely unknown. Finally, the immunological mechanisms underlying various types of liver diseases are not explored in this issue; hopefully, another special issue for clinical liver immunology will discuss these mechanisms in the future.

1 Racanelli V, Rehermann B. The liver as an immunological organ. Hepatology 2006; 43 : S54-S62.

2 Gao B, Jeong WI, Tian Z. Liver: an organ with predominant innate immunity. Hepatology 2008; 47: 729-736.

3 Sheth K, Bankey P. The liver as an immune organ. Curr Opin Crit Care 2001; 7: 99-104.

4 Bogdanos DP, Gao B, Gershwin ME. Liver immunology. Compr Physiol 2013; 3: 567-598.

5 Crispe IN. The liver as a lymphoid organ. Annu Rev Immunol 2009; 27: 147-163.

6 Robinson M, Harmon C, O'Farrelly C. Liver Immunology and its role in inflammation and homeostasis. Cell $\mathrm{Mol}$ Immunol 2016 (in issue).

7 Horst A, Neumann K, Diehl L, Tiegs G. Modulation of liver tolerance by conventional and nonconventional antigen presenting cells and regulatory innate immune cells. Cell $\mathrm{Mol}$ Immunol 2016 (in issue).

8 Grakoui A, Crispe IN. Presentation of hepatocellular antigens. Cell Mol Immunol 2016; 13: 292-299.

9 Zhou Z, Xu MJ, Gao B. Hepatocytes: a key cell type for innate immunity. Cell Mol Immunol 2016; 13: 300-314.

$10 \mathrm{Ju}$ C, Tacke F. Hepatic macrophages in homeostasis and liver diseases: from pathogenesis to novel therapeutic strategies. Cell Mol Immunol 2016; 13: 315-326.

11 Peng H, Wisse E, Tian Z. Liver natural killer cells: subsets and roles in liver immunity. Cell Mol Immunol 2016; 13: 267-275.

12 Bandyopadhyay K, Marrero I, Kumar V. NKT cell subsets as key participants in liver physiology and inflammation. Cell Mol Immunol 2016 (in issue).

13 Knolle P, Wohlleber D. Immunological functions of liver sinusoidal endothelial cells. Cell Mol Immunol 2016 (in issue).

14 Shuai Z, Leung M, He X, Zhang W, Yang G, Leung $P$ et al. Adaptive immunity in liver. Cell Mol Immunol 2016 (in issue).

15 Wisse E, van't Noordende JM, van der Meulen J, Daems WT. The pit cell: description of a new type of cell occurring in rat liver sinusoids and peripheral blood. Cell Tissue Res 1976; 173: 423-435.

16 Peng $\mathrm{H}$, Jiang X, Chen Y, Sojka DK, Wei H, Gao $X$ et al. Liver-resident NK cells confer adaptive immunity in skin-contact inflammation. J Clin Invest 2013; 123: 1444-1456.

17 Taniguchi H, Toyoshima T, Fukao K, Nakauchi $\mathrm{H}$. Presence of hematopoietic stem cells in the adult liver. Nat Med 1996; 2: 198-203. 